\title{
Correlative Imaging in the Study of Oxidative Stress in Two Animal Models: Experimental Allergic Encephalomyelitis and Diabetic Retinopathy
}

\author{
E. Ann Ellis ${ }^{*}$, John R. Guy ${ }^{* *}$, and Maria B. Grant ${ }^{* * *}$ \\ *Microscopy and Imaging Center, MS 2257, Texas A \& M University, College Station, TX 77843 \\ ** Dept. of Ophthalmology, ${ }^{* * *}$ Dept. of Pharmacology \& Therapeutics, College of Medicine, \\ University of FL, Gainesville, FL 32610
}

Animal models play an important role in biomedical research as models for human disease processes. Often these studies are based on biochemical analyses and/or histopathology studies during chronic or terminal stages of the disease. Since animal models are such an expensive part of biomedical research, every effort should be made to obtain maximum data from all animals. Our work with experimental allergic encephalomyelitis (EAE) and diabetic retinopathy utilized multiple imaging techniques and correlative methods with time course studies to maximize the data obtained from all experimental and control animals. EAE, a frequently used model of multiple sclerosis, was induced in strain 13 guinea pigs and used for studies of the role of oxidative stress in this demyelinating disease. A spontaneous model of adult onset diabetes, the BBZ/Wor rat, and galactose induced diabetes in nitric oxide synthase (NOS) knockout mice provided reliable models for studying the role of oxidative stress in diabetic retinopathy from the onset of hyperglycemia.

Oxidative stress results when free radicals or reactive oxygen species are produced in excess of free radical scavengers such as ascorbic acid, $\alpha$-tocopherol, or antioxidant enzymes (superoxide dismutase, catalase, glutathione peroxidase). Direct detection of reactive oxygen species has been difficult because these species are short lived and catalyzed rapidly by scavenging systems. The cerium NADH/NADPH oxidase methodology of Briggs and Karnovsky [1] has been used for localization of the free radical derived oxidant, hydrogen peroxide $\left(\mathrm{H}_{2} \mathrm{O}_{2}\right)$ and has been an important part of our studies of cellular/subcellular localization of free radical generating systems in conjunction with local pathology. This methodology is also compatible with post embedding colloidal gold labeled immunocytochemistry.

Studies of optic neuritis/multiple sclerosis in EAE employed magnetic resonance imaging (MRI), morphometric analysis of demyelination [2], cytochemical localization of sites of $\mathrm{H}_{2} \mathrm{O}_{2}$ production [3], and immunocytochemical localization of sites of serum albumin extravasation (a marker for disruption of the blood retinal barrier). The clinical progress of optic neuritis in optic nerves of strain 13 guinea pigs was documented by MRI without and with gadolinium-DTPA (Gd-DTPA) contrast [4]. The MRI studies were similar to the procedures used to help diagnose and follow optic neuritis in human patients. Age matched animals were imaged by MRI prior to induction of EAE and then every three to four days. Several hours after the last MRI images were done, the animals were euthanized and the eyes and adjacent optic nerves to the chiasm were removed immediately, fixed in cold 5\% acrolein in $0.1 \mathrm{M}$ sodium cacodylate buffer $(\mathrm{pH} 7.4)$ for one hour and then washed 4X15 minutes in $0.15 \mathrm{M}$ cacodylate buffer plus 5\% sucrose and 1\% dimethyl sulfoxide (DMSO). Specimens could then be held in buffer wash in the refrigerator until localization of NADH/NADPH oxidase was done by the cerium based method of Briggs et al. [1]. After localization of $\mathrm{NADH} / \mathrm{NADPH}$ oxidase specimens were then dehydrated through an ethanol series and infiltrated 
and embedded in epoxy resin. Histopathology was studied in $2 \mu \mathrm{m}$ thick sections stained with toluidine blue; ultra-thin sections were cut median longitudinally through the optic nerve head and consecutive pieces of optic nerve. Time course studies using MRI and transmission electron microscopy showed correlation of gadolinium enhancement with inflammatory cell infiltrates and that disruption of the blood retinal barrier preceded widespread demyelination [5]. Morphometric analysis of Gd-DTPA leakage in optic nerves was done on MRI images from antioxidant drug treated and vehicle treated animals and compared to quantitation of cerium perhydroxide (marker for $\mathrm{H}_{2} \mathrm{O}_{2}$ localization) for the same areas of optic nerve [6].

Hyperglycemia is the primary initiating factor for all types of diabetic vascular complications including diabetic retinopathy and increased oxidative stress has been implicated in the development of vascular complications. In diabetic hyperglycemia there is increased flux of glucose via the sorbital pathway that results in increased cytosolic NADH/NAD ${ }^{+}$increased superoxide described as hyperglycemia-induced pseudohypoxia. Cytochemical localization of NADH oxidase in retinas of non-insulin dependent diabetic BBZ/Wor rats, demonstrated localization of $\mathrm{H}_{2} \mathrm{O}_{2}$ in endothelial cells and post embedding immunocytochemical localization of serum albumin leakage showed disruption of the blood retinal barrier. Results of these two correlative techniques support a role for hyperglycemia induced oxidative stress in vascular complications of diabetic retinopathy [7]. Time course studies using cytochemical localization of NADH oxidase and post embedding immunocytochemical localization of inducible NOS and nitrotyrosine demonstrated increased production of nitric oxide and increased inactivation of nitric oxide which coincided with the onset of hyperglycemia [8]. These results suggest that oxidative stress contributes to retinal damage in diabetes from the onset of hyperglycemia.

Correlative studies of $\mathrm{NADH}$ oxidase cytochemical localization and immunocytochemical localization of nitrotyrosine in galactose induced diabetes in wild type, endothelial NOS knockout, and inducible NOS knockout mice demonstrated that inducible NOS is the source of nitric oxide in increased production and increased inactivation of nitric oxide in diabetic retinopathy [9].

Our studies on these two animal models demonstrate the importance of using multiple imaging techniques, correlative methods and time course studies to document and interpret early events in the pathology of chronic diseases.

\section{References}

[1] R. T. Briggs et al., J. Cell Biol. 67(1975)566.

[2] J. Guy et al., J. Comp. Neurol. 287(1989)446.

[3] J. Guy et al., Arch. Ophthalmol. 108(1990)1614.

[4] J. Guy et al., Ophthalmol. 97(1990)601.

[5] J. Guy et al., Ophthalmol. 99(1992)720.

[6] J. Guy et al., Ophthalmic Res. 25(1993)253.

[7] E. A. Ellis et al., Free Rad. Biol. Med. 24(1998)111.

[8] E. A. Ellis et al., Nitric Oxide 6(2002)295.

[9] E. A. Ellis et al., Invest. Ophthalmol. Vis. Sci. 43 Suppl.(2002)Abstr. 2120. 